\title{
Alumnos sobresalientes: visión en torno a la atención que reciben
}

\author{
Pedro Covarrubias Pizarro
}

Universidad Autónoma de Chihuahua

\section{Resumen}

Se presentan los resultados obtenidos en un trabajo de investigación evaluativa, en donde se rescataron las opiniones de las y los alumnos identificados como sobresalientes y que fueron atendidos con el modelo de atención implementado por la Secretaría de Educación Pública, estipulado en la Propuesta de Intervención Educativa para alumnas y alumnos con Aptitudes Sobresalientes, en el ciclo escolar 2007-2008. La investigación buscó indagar cuatro aspectos relacionados con la implementación de la Propuesta: (1) la evaluación general que se tiene del trabajo realizado; (2) la valoración de los procesos llevados a cabo; (3) los resultados obtenidos; y (4) la visualización del futuro con respecto a la atención educativa. Se empleó una metodología de corte mixto con predominancia en lo cualitativo y considera diferentes momentos, actores e instrumentos para la recuperación de los datos y su posterior análisis. Participaron en la evaluación el personal de educación especial del subsistema estatal del estado de Chihuahua, padres y madres de familia de niñas y/o niños sobresalientes, maestros de grupo regular, alumnos identificados con el modelo de intervención propuesto y un grupo de expertos nacionales en el tema de las altas capacidades. En este trabajo se presentan exclusivamente los hallazgos encontrados bajo la visión y valoración de las $y$ los alumnos sobresalientes.

Palabras clave: alumnos sobresalientes, propuesta educativa, investigación evaluativa.

\section{Introducción}

A fin de atender a las y los alumnos que poseen aptitudes sobresalientes en un marco de equidad y atención a la diversidad, en 1987 surgió en México el Proyecto para niños con Capacidades y Aptitudes Sobresalientes $^{1}$-Proyecto CAS-, el cual dejó de operar a mediados de los años 90'. En el

1 En lo sucesivo se empleará Propuesta de AS, para referirse a la Propuesta de intervención: atención educativa a alumnos y alumnas con aptitudes sobresalientes, y evitar el título extenso del documento. La abreviatura AS significa Aptitudes Sobresalientes. 
2007, se retomó la atención de esta población y se implementó la Propuesta de Intervención: Atención educativa a alumnos y alumnas con aptitudes sobresalientes(SEP, 2006a), como un Programa Nacional destinado a atender integralmente a esta población. A seis años de su implementación, este programa no ha sido evaluado sistemáticamente en la entidad, por tanto no se cuenta con resultados que permitan realizar ajustes en su operatividad y valorar el impacto que ha tenido.

La investigación abordó como objeto de estudio a la evaluación de la Propuesta de AS y la pregunta de investigación fue: ¿cuáles son los resultados de la implementación de la Propuesta de intervención: atención educativa a alumnas y alumnos con aptitudes sobresalientes? El propósito de esta comunicación es presentar la visión que tienen las y los alumnos identificados como sobresalientes con respecto al trabajo que se ha realizado con el modelo de la Propuesta de AS.

\section{Método}

Dada la naturaleza del objeto de estudio, la investigación empleó una metodología mixta con predominancia en el dato cualitativo. De acuerdo con Hernández (2010), este tipo de metodología reúne un conjunto de procesos sistemáticos, empíricos y críticos que facilitan la evaluación, la recolección de datos y su análisis, así como un mejor entendimiento de la realidad estudiada.

El estudio se sustentó prioritariamente en el modelo de investigación evaluativa planteado por Stake (2006) denominado
Evaluación comprensiva. Stake, señala que la evaluación comprensiva surge en el marco del paradigma cualitativo, por tanto en ese sentido es interpretativa y sensible o receptiva a la actividad en el tiempo; rescata las percepciones y voces de las personas que retratan la experiencia humana.

Considerando estos principios se diseñó y aplicó un modelo de evaluación que integró cuatro componentes: (1) evaluación general, (2) evaluación de procesos, (3) evaluación de resultados y (4) la institucionalización. A la par de estos componentes se establecieron cuatro criterios de calidad: pertinencia, coherencia, relevancia y eficacia.

Con el modelo de evaluación se propusieron diferentes técnicas e instrumentos (Álvarez-Gayou, 2006; Balcazar, 2006): cuestionario abierto para el personal de educación especial; grupos focales para maestros de grupo regular, padres y madres de familia, y con alumnos identificados como sobresalientes. También se aplicaron entrevistas semiestructuradas al grupo de expertos nacionales en el campo de las altas capacidades. Se documentaron $d a-$ tos numéricos relacionados con la estadística de alumnos, así como el análisis de los presupuestos financieros otorgados por el Gobierno Federal, para la implementación de la Propuesta de AS.

Hablando específicamente del grupo conformado por niñas y niños sobresalientes -del cual se reportan los hallazgos-, se llevó a cabo con la participación de 10 alumnos en total, 4 mujeres y 6 hombres. Los participantes pertenecían a diferentes escuelas primarias y se ubicaban en 
diferentes grados escolares desde $1^{\circ}$ hasta $6^{\circ}$ grado. Las aptitudes sobresalientes que poseen corresponden al área intelectual preferentemente, algunos artísticos, otros creativos y una alumna socioafectiva. En la investigación participaron un total de 122 informantes.

La organización de los datos obtenidos, se dio acorde a los cuatro componentes del modelo de evaluación. Para el análisis de los datos cualitativos se empleó el programa Atlas Ti. La conformación de categorías se trabajó hasta la reducción de datos para obtener unidades significativas y manejables (García, 1992); finalmente se emitieron algunas conclusiones donde se conjuntó la visión de todos los actores participantes.

\section{Resultados}

\section{La visión de las y los alumnos sobre- salientes}

Los resultados que se presentan a continuación describen exclusivamente la opinión y valoración que realizaron los usuarios directos de la Propuesta de AS, es decir las y los niños identificados con aptitudes sobresalientes. La organización de los hallazgos corresponde a los cuatro componentes del modelo de evaluación. Se incorporan algunos testimonios para enriquecer el trabajo.

\section{Categoría 1. Evaluación general}

Un componente importante de la evaluación de programas educativos es conocer el nivel de satisfacción de los usuarios, que en este caso son las y los alumnos. Con los participantes se abordó la primera categoría a partir de la pregunta generadora ¿cuál ha sido tu experiencia al ser identificado como niño o niña con aptitudes sobresalientes? Este cuestionamiento permitió indagar al respecto de qué cosas les agradan y cuáles no.

Las opiniones que expusieron las y los alumnos, permitieron identificar dos subcategorías importantes: por una parte se refleja la autosatisfacción vista como un logro y orgullo personal, que les permite ser reconocidos y valorados por sus potenciales y capacidades personales. Se fortalece una identidad como niño o niña sobresalientes que permite el saberse sobresaliente e inteligente, con posibilidades de aprendizaje diferentes a las del resto de sus compañeros. Expresiones como: "Se siente bonito ser sobresalientes", "sé que soy sobresaliente y estoy orgullosa de mí misma", o "me gusta ser inteligente", son testimonios que avalan esta condición.

La segunda subcategoría correspondiente a la experiencia de ser un estudiante con esta condición, guarda relación con la importancia que los alumnos/as sobresalientes sienten, satisfacción por poder colaborar con los demás compañeros del grupo. Para ellas y ellos es importante y un beneficio de su condición, saber que pueden apoyar en diferentes tareas académicas a los alumnos de sus grupos. Se pone de manifiesto que su potencial es utilizado para establecer trabajo de acompañamiento en la realización de tareas, explicaciones personalizadas y un aprendizaje mutuo. El siguiente testimonio resume el sentir de varios de los participantes: "tanto yo aprendo de mis compañeros, como ellos aprenden de mi”.

Con un menor número de menciones 
pero no por ello menos importante, se puede también identificar un aspecto negativo del ser sobresaliente; algunos alumnos y alumnas hacen mención a ser víctimas del abuso que pueden sufrir por su condición, ya que dentro de las aulas existen compañeros que les piden las tareas o les copian los ejercicios de trabajo. En el siguiente testimonio se puede dar cuenta de lo anterior: “...solo a veces alguien se aprovecha de mí, por mi inteligencia. Es molesto de vez en cuando".

\section{Categoría 2. La implementación}

Con las y los alumnos se exploraron los puntos relacionados con el proceso de implementación de la Propuesta de AS, tales como la identificación, evaluación e intervención. Las preguntas que dieron pauta a las opiniones fueron: ¿cómo fuiste identificado?, ¿cómo fuiste valorado? y ¿de qué forma trabajan contigo? Los hallazgos que se presentan están organizados en estas tres fases del proceso de implementación:

I. Una de las condiciones particulares en la atención de alumnas y alumnos que presentan necesidades específicas es involucrar al individuo en el proceso de identificación, con el objeto de sensibilizar sobre su condición. Ya sea un alumno con una discapacidad o bien con aptitudes sobresalientes, es necesario que se le expliquen los procesos de identificación y valoración a los cuales será sometido. En este caso en particular se indagó en el grupo focal los mecanismos por los cuáles fueron identificados y el conocimiento que tenían de ello.
Los resultados arrojan resultados reveladores, por una parte existe una gran mayoría de casos en que las y los alumnos desconocen cómo fueron identificados, ya que no se les informó de los mecanismo, ni de las pruebas utilizadas para integrarlos al grupo de AS. Se puso de manifiesto que existen ciertas creencias al respecto que nada tienen que ver con los procesos estipulados en la Propuesta de AS. Algunos de los alumnos piensan que fueron incluidos porque ya venían identificados desde preescolar, otros suponen que fueron identificados por sus calificaciones, e incluso hubo una participación que supone que el lugar en el grupo de niños sobresalientes fue heredado. Aquí el testimonio: "Mi hermano no estaba y cuando a él le dijeron, también me empezaron a ver a mí, y cuando salió mi hermano me dejaron el lugar a mí, y vieron que si era sobresaliente".

II. En la parte de evaluación psicopedagógica, los resultados tomaron la misma dirección que la detección, es decir un desconocimiento tanto de los procedimientos como de los instrumentos. Existen algunos alumnos y alumnas que dicen que fueron sometidos a varias pruebas, pero no saben con exactitud cuáles o para qué servían. Solo hacen referencia a trabajar con unas pruebas que tenían problemas, que utilizaban algunos cubos o que completaban figuras. Podría suponerse que se están refiriendo a las prueba de la Escala de Weschler para evaluar el coeficiente intelectual. Finalmente 
varios alumnos simplemente dijeron: "no sé cómo".

III. Con la finalidad de conocer el trabajo que se realizó en las escuelas con las y los alumnos sobresalientes, se indagó con los participantes el tipo de actividades que se realizan, quiénes son los responsables de atenderlos, y cuáles son los espacios donde se lleva a cabo el trabajo.

Con respecto al tipo de actividades que se llevaron a cabo para ser atendidos, la mayoría de los participantes hicieron mención del uso de proyectos como la estrategia predominante. Generalmente son proyectos temáticos -artes, ciencias naturales o ciencias- $y$ fueron dirigidos exclusivamente para los alumnos con AS, no con la totalidad del grupo al que pertenecen. Esta modalidad es coordinada principalmente por la maestra del servicio USAER y no se menciona la participación de las o los maestros del grupo regular. En el siguiente testimonio se da cuenta de ello: "Estamos realizando el proyecto de USAER y AS sobre animales"

Otra de las actividades que las y los alumnos reportaron como frecuentes fueron las salidas fuera de la escuela. Este tipo de actividades fueron de las más motivantes para las y los estudiantes, principalmente cuando se visitan museos interactivos.

Otra de las actividades que mencionaron los alumnos fue fungir como monitor dentro del aula. Esta estrategia es utilizada con la finalidad de que los alumnos sobresalientes puedan favorecer el aprendizaje de sus compañeros. Testimonio: "Les explico a mis compañeros, les enseño".

Una de las estrategias que se deberían proponer para las y los alumnos sobresalientes de acuerdo con la Propuesta de AS, es la ampliación horizontal o la ampliación vertical, que guarda relación con el manejo de contenidos acelerados o de mayor profundidad. En este caso únicamente un alumno mencionó que se lleva a cabo este tipo de intervención. "Me gusta cuando me llevan al salón de USAER y me ponen sumas de cuarto y quinto año; estoy en primer año y si las hago". Se presenta como una actividad que realizan dentro del aula de USAER y no como una adecuación que se realice cotidianamente en el aula regular.

En general puede concluirse que existe poca diversidad en las estrategias de intervención que se llevan a cabo. En voz de los participantes se rescata la valoración general que realizan del trabajo desde su propia óptica y en este sentido existe una mayoría que opinan que si bien es cierto les agrada lo que realizan en la escuela, existe una fuerte inquietud de que sean trabajos más dinámicos, innovadores, motivadores y con mayores retos para ellos. Existe una fuerte necesidad de recibir mejores actividades que logren despertar su curiosidad, interés y sobre todo el reto intelectual. Algunos testimonios de ello son los siguien- 
REVISTA DE INVESTIGACIÓN EDUCATIVA DE LA REDIECH N. 8

ISSN: 2007-4336

tes: “...queremos cosas que atrapen la atención con educación"; "proyectos más interesantes"; "que sea más dinámico, más interesante".

Cabe mencionarse que un alumno manifestó que sentían que no se estaba haciendo nada novedoso con ellos, o que hacían cosas demasiado fáciles. Su testimonio así lo dice: "No hemos hecho mucho y nada novedoso".

La voz de las y los alumnos fue un fuerte indicador del trabajo que realiza la escuela regular y los servicios de educación especial en torno a la intervención. Ellos son los portadores de una inquietud manifiesta en donde se deja establecido lo que quisieran aprender y desarrollar como parte de un programa para alumnos de altas capacidades. Al ser sobresalientes, pueden identificar sus propias necesidades de aprendizaje y lo esperan recibir de la escuela: una formación adecuada para su potencial.

IV. Como parte de la implementación de la Propuesta de AS, también indagó con las y los alumnos con aptitudes sobresalientes, el espacio en donde reciben la intervención y por parte de quién la reciben. Solo existen dos alternativas: se trabaja con ellas y ellos dentro del aula regular o bien en el aula de USAER. No existe una constante que refleje el espacio de intervención; las y los alumnos refieren ser atendidos en ambos espacios. Lo que sí es un hecho predominante es que la atención recae en el trabajo del servi- cio de educación especial, y no en los maestros de grupo.

V. Un último aspecto que se indagó fue el trabajo que desarrollan las y los padres de familia como tarea complementaria en la intervención. En este caso las y los niños no perciben una vinculación entre lo que realizan en la escuela regular y la continuidad en sus hogares. Desde la óptica de los alumnos el trabajo que se realiza en casa es una formación extracurricular relacionada con otro tipo de actividades. Todas y todos los alumnos participan activamente en clases extracurriculares relacionadas con el deporte o las artes. Algunos de ellos toman cursos específicos de inglés o robótica, pero en su mayoría reportan realizar deportes como futbol americano, gimnasia, karate o danza hawaiana.

Categoría 3. Los resultados y logros obtenidos

A través de la pregunta generadora ¿qué cosas has logrado como niño o niña sobresaliente?, se exploró la visión que tienen las y los alumnos de logros y resultados obtenidos.

En la voz de los participantes, manifestaran como logros haber obtenido beneficios de corte académico, es decir, relacionan directamente los beneficios con el hecho de adquirir más conocimientos y habilidades, así como la adquisición de mejores y nuevos aprendizajes. Las y los alumnos consideraron que el programa de intervención ha incidido en su formación académica con 
elementos que les han permitido mejorar su rendimiento escolar. Testimonios: "He aprendido más, varios proyectos extras, cosas que mis compañeros no lograrían hasta ahora, no se me dificultan cosas como a los demás"; "He mejorado las cosas que sé". En la mayoría de los casos, el punto de referencia para valorar los logros obtenidos, fue la comparación con los compañeros de grado y grupo.

Otras de las opiniones aisladas que se dieron con respecto a los logros, fue el haber obtenido el reconocimiento de sus compañeros del grupo, así como el de los maestros de la escuela. Este reconocimiento se manifiesta cuando los alumnos les piden mayores apoyos, asesorías, o incluso cuando las maestras les dejan la responsabilidad de "cuidar" al grupo o dirigir la clase.

La última manifestación de logro que se puso en evidencia, guardó relación nuevamente con el aspecto académico, pues algunas de las y los alumnos manifiestan que el haber estado atendidos por la maestra de AS, les ha permitido participar y ganar premios y concursos académicos. Testimonio: "En un concurso académico me saque una medalla de oro".

\section{Categoría 4. Institucionalización y} generalización

Para indagar la posibilidad de generalizar e institucionalizar la atención educativa por medio de la Propuesta de AS, se preguntó a los alumnos ¿Te gustaría que esto continuara? y ¿cómo te ves el futuro?

Al indagar si les gustaría que la atención continuara hubo consenso al respecto, ya que todas y todos manifestaron que sí; sin embargo pusieron en evidencia que esa atención debería continuar con ciertas condiciones, como por ejemplo con actividades de mayor interés, innovación y motivación; demandan nuevamente que se implementen actividades que impliquen retos y que sean divertidas para ellos. Solo algunos alumnos (los de grados superiores) manifestaron su inquietud y preocupación por la continuidad de la atención en la secundaria, ya que saben que en la entidad existen muy pocas escuelas de ese nivel, que dan atención a los sobresalientes.

La visión del futuro que tienen sobre ellos mismos en algunos años resultó ser sumamente positiva, ya que todas y todos ellos se visualizan como profesionistas en diferentes áreas. La lista de profesiones incluyó "bailarines profesionales, gastrónomos, investigadores de la NASA, psicólogos, arquitectos, inventores o científicos; astronautas, criminólogos, médicos, contadores, espías, escritores", entre otras.

\section{Conclusiones}

La Propuesta de AS, no ha cubierto del todo las necesidades de los alumnos sobresalientes debido a los mecanismos de operatividad que se han implementado.

Las y los alumnos reconocen los beneficios de ser atendidos como alumnos sobresalientes y realizan una estrecha relación con logros de orden académico.

Se percibe una fuerte necesidad de recibir un tipo de intervención más motivante e interesante para ellos, ya que demandan creatividad, esfuerzo y mayores retos que los que están recibiendo. 


\section{Las y los alumnos desconocen los meca- Referencias}

nismos de identificación y evaluación con los cuales fueron seleccionados.

No existe un patrón regular en los procedimientos de intervención que cada alumno recibe. Existe una desvinculación entre los servicios de educación especial y la escuela regular, para dar la intervención educativa.
Álvarez-Gayou, J. L. (2006). Cómo hacer investigación cualitativa. Barcelona: Paidós Educador.

Balcazar Nava, G.-A. G. (2006). Investigación cualitativa. Universidad Autónoma del Estado de México.

Gagné, F. (2010). Construyendo talentos a partir de la dotación: breve revisión del MDDT 2.0. En D. V. Valdez Sierra, Desarrollo y Educación del talento en Adolescentes. Nivel Medio Básico y Medio Superior (págs. 64-78). Guadalajara: Universitaria.

Hernández Sampieri, et al (2010). Metodología de la investigación. México, D.F. Mc Graw Hill.

SEP, (2006a). Propuesta de intervención: Atención educativa a alumnas y alumnos con aptitudes sobresalientes. México: Secretaría de Educación Pública

SEP. (2006). Orientaciones para el funcionamiento de los servicios de educación especial. México: Secretaría de Educación Pública.

Stake, R. (2006). Evaluación Comprensiva y evaluación basada en estándares. España: GRAÓ. 